\title{
Influence of Short Video Watching Behaviors on Visual Short-Term Memory
}

\author{
Mengyu Zheng \\ School of Physiology, Pharmacology and Neuroscience, University of Bristol, Bristol, BS8 1QU, UK, \\ ai20279@bristol.ac.uk
}

\begin{abstract}
This study examined the influence of an average daily viewing time of short videos on viewers' visual short-term memory. Participants were asked to either watch short videos or rest before taking a secondary pattern recognition task that tests their visual short-term memory. Results showed that the participants' ability to memorize patterns and respond quickly was significantly impaired by short video watching behaviors. We conclude that short videos do have a negative impact on visual short-term memory, yet its mechanisms were not thoroughly investigated in this study.
\end{abstract}

Keywords: visual short-term memory, short videos

\section{INTRODUCTION}

Short video apps such as Douyin (Chinese version of TikTok) features videos that are usually 15 or 60 seconds in length[1]. While genres and contents vary across different videos, these apps share similar recommendation algorithms that create a reward loop showing videos a viewer is interested in [1]. On average, users spend 24.5 minutes per day on short video apps, with Douyin being the most popular of its kind.

As short video apps increasingly gain their popularity, a concern for the impact of short videos on memory has also risen among their users. While much research has been done on visual short-term memory (VSTM) or the effects of other media types on this aspect, studies focusing on short videos are still scarce. This research investigated how short videos affect viewers' accuracy and response time in a secondary pattern recognition task and, thereby, the viewers' VSTM.

External sensory inputs, such as visual stimuli, are immediately registered within the appropriate sensory dimension when presented but are temporally fleeting and prone to decay [2]. Further processing is required to consolidate visual registration into visual short-term memory (VSTM) [3], which lasts considerably longer than the former. Factors that have a distracting effect on VSTM are known as distractors [4].

Previous studies have proven that visual structural features can elicit orienting response (OR), an involuntary response triggered by changes in the environment. Viewers of video cuts or movement showed decreases in the alpha frequency [5] and behavioral response (such as turning eyes toward the source of visual stimulus) [6], which are all characteristics for OR. However, how or whether this change in attention resulting from OR affects VSTM is less thoroughly studied. Ohman [7] suggested integrating OR with the limited-capacity attention model, where OR is the selecting process for information to enter a limitedcapacity processing channel and consequently picked up by conscious awareness. Lavie [8], on the other hand, proposed that physical distinction, although necessary, is not sufficient to initiate selective processing. She suggested that information selection only initiates when the capacity limit is met. Perceptual selection is, therefore, the consequence of allocating a limited load of attention. Thus, the capacity limitation for the transition from perception to memory may orient from the following three aspects: 1 . Perceptual limitations that interfere with the amount of stimulus received; 2 . Attentional limitations relating to OR; and 3. Processing capacity that pose limits to viewers' ability to process and storage information before subsequently transform it into memory [9].

By conducting secondary tests, A. Lang [10] and Friestad and Thorson [11] have shown that when using structural features (e.g., television cuts, videotaped lectures.) as distractors, memory for the specific information (stimulus) that follows them can be interfered. These interferences can either improve or 
damage the VSTM depending on the processing capacity available to be allocated when a stimulus occurs. Specifically, the difficulty of the prior content and its relevance to the stimulus are two determining factors: viewing difficult or irrelevant distractors is suggested to negatively affect information processing afterwards; memory for stimulus presented before the distractors are not affected. On the other hand, Newhagen and Reeves [12] showed that compelling, emotional visual information could have a negative and retroactive effect on VSTM.

Based on the above previous research, we adopted a similar experimental procedure using short videos as distractors and a secondary pattern recognition test as a mean to assess subjects' VSTM. We propose a research hypothesis that viewers' VSTM is negatively impacted by an average daily viewing time of short videos. The design requires the experimental group to watch an average daily viewing time of short videos whereas the control group does not, after which both group perform the identical VSTM test. If the research hypothesis is valid, subjects who watched short videos should observe lower performances than their control group counterpart. Performances is measured in terms of response time, answering rate and correctness rate.

\section{METHODS}

\subsection{Participants}

10 participants $(2$ males, 8 females; age mean $=19.6$ yrs, $\mathrm{SD}=0.8$ ) participated in the study. All participants reported normal or corrected-to-normal vision and were right-handed. None of the participants reported having or have had any neurological or psychiatric disorder. All of them were native Mandarin speakers. Participants' years of education and short video viewing habits (daily viewing time and most frequently used app) were also collected during pre-screening via self-report.

\subsection{Experimental procedure}

At the beginning of the experiment, subjects were informed of the experimental procedure (fig.1), and they each signed a consent form. The age, gender, handedness, vision, level of education, self-reported short video viewing time per day of participants were recorded during pre-screening.

Five of the participants were randomly selected to undergo five sets of repeated experimental blocks, during which 5 min of TikTok videos were viewed followed by ten pattern recognition VSTM questions (i.e., a total of 25 min video-watching and 50 VSTM questions). The remaining 5 participants (control group) completed the same experimental procedure but did not watch any videos. An experimenter closely monitored the control group to ensure they did not engage in any attentiondemanding activities (e.g., reading, watching videos, replying to messages). All videos were presented from participants' personal mobile phones due to constraints in experimental conditions.

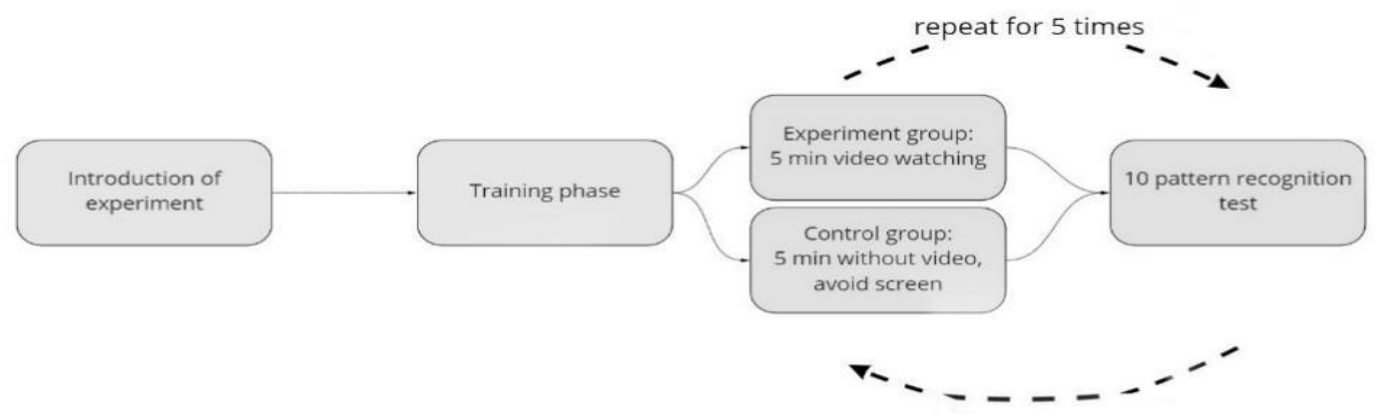

Fig.1: Experimental procedure.

\subsection{Short Video Distractors}

Short videos are used as distractors and are either 15 $\mathrm{s}$ or $60 \mathrm{~s}$ in maximum length. As algorithms of short video apps render considerable variance of recommendation among users, we asked participants to watch videos from their most frequently used app to imitate their daily viewing experience maximally. Alternatively, if a participant does not have a habit of watching short videos, we asked them to view videos from a blank account on Douyin (the most popular short video app in Mainland China) to ensure a relatively unbiased video selection.

\subsection{VSTM test}

Following each video viewing period (experimental group) or rest period (control group) of $5 \mathrm{~min}$, a pattern recognition VSTM test was performed on each participant as a secondary task (fig. 2) via Zoom and 
PsychoPy. Before each pattern (stimulus) display, a white fixation cross appeared for $0.5 \mathrm{~s}$ at the centre of the grey background. This was then replaced by the stimulus pattern. After the pattern was shown for $0.5 \mathrm{~s}$, there was another identical fixation for another $0.5 \mathrm{~s}$. Participants then tried to identify the shown pattern from 4 choices (fig. 3) available on screen for $2 \mathrm{~s}$, although their response time could exceed 2s. Participants were instructed to pay close attention to the test and verbalize their choice as quickly as possible. Both groups had their response and verbalized response time recorded for further analysis.

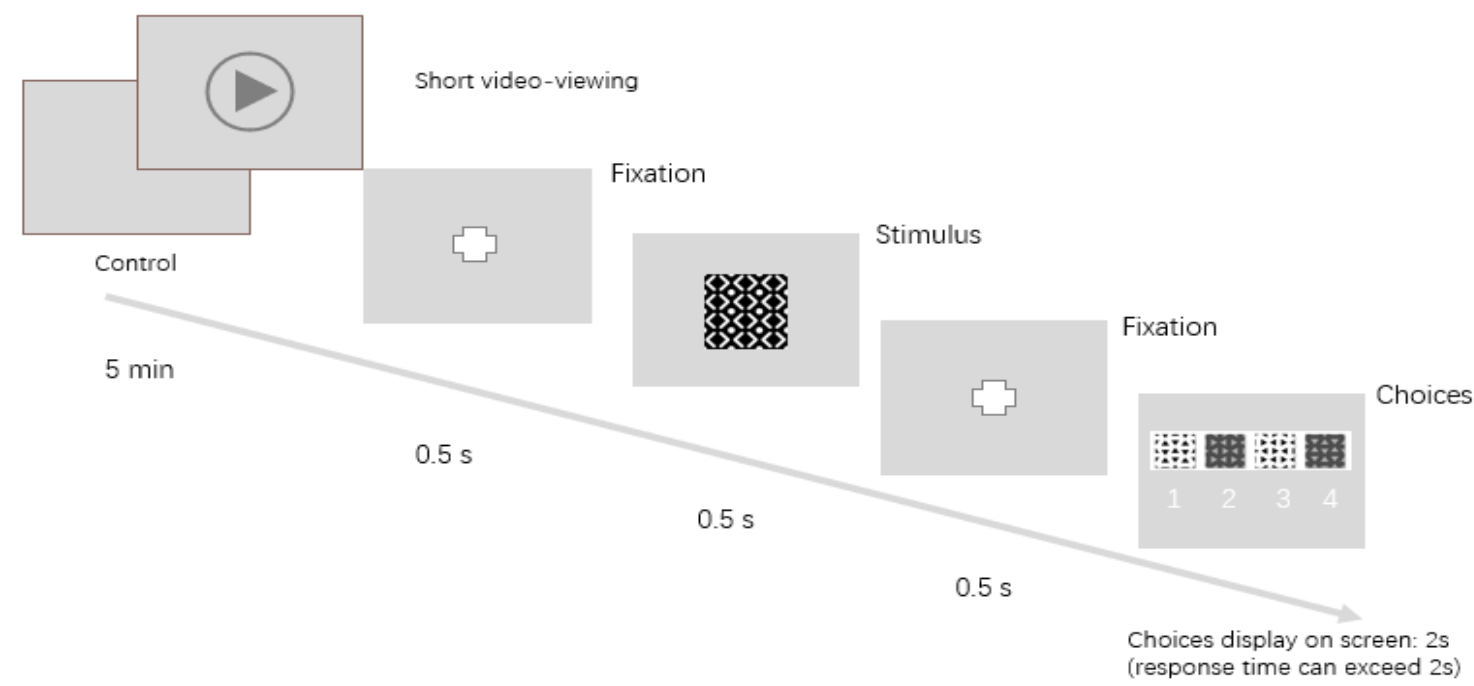

Fig. 2: Secondary pattern-recognition VSTM test.

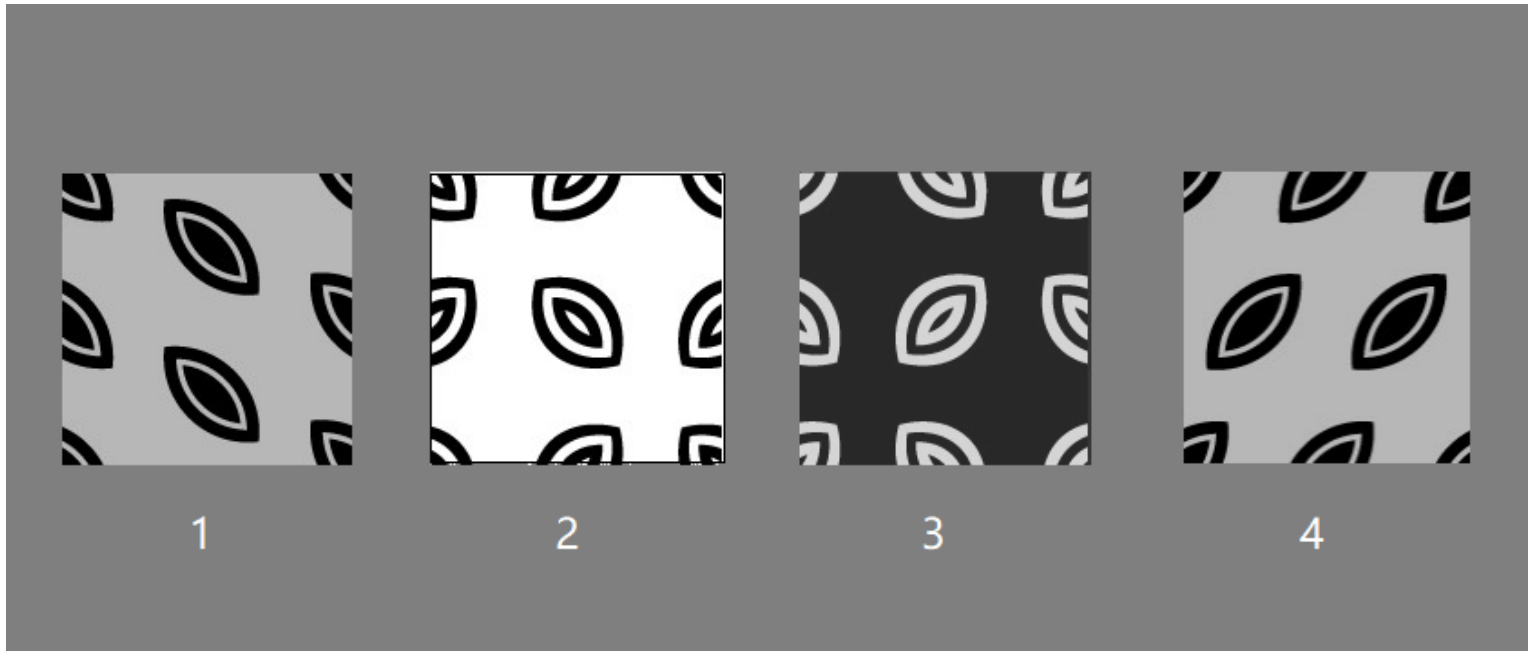

Fig. 3 Example of pattern choices in VSTM test: the four choices in one pattern recognition question are matched to share visual similarities while also having differences in both pattern and colour. One of four choices is the correct pattern shown as the stimulus. Choices are numbered to allow participants to verbalize their answers.

Since verbalized response time required manual recording, we conducted a single-blind experiment where the experimenter who did the recording was kept unaware whether a subject belongs to the experimental group or control group to prevent biased data.

\section{RESULT}

Participants in both groups were highly accurate in the secondary pattern-recognition test. The control group had a mean answering rate of $95.2 \%$ and a mean correctness rate of $84.8 \%$, whereas the data for the experimental group were $87.6 \%$ and $76.0 \%$, respectively (fig. 4). 


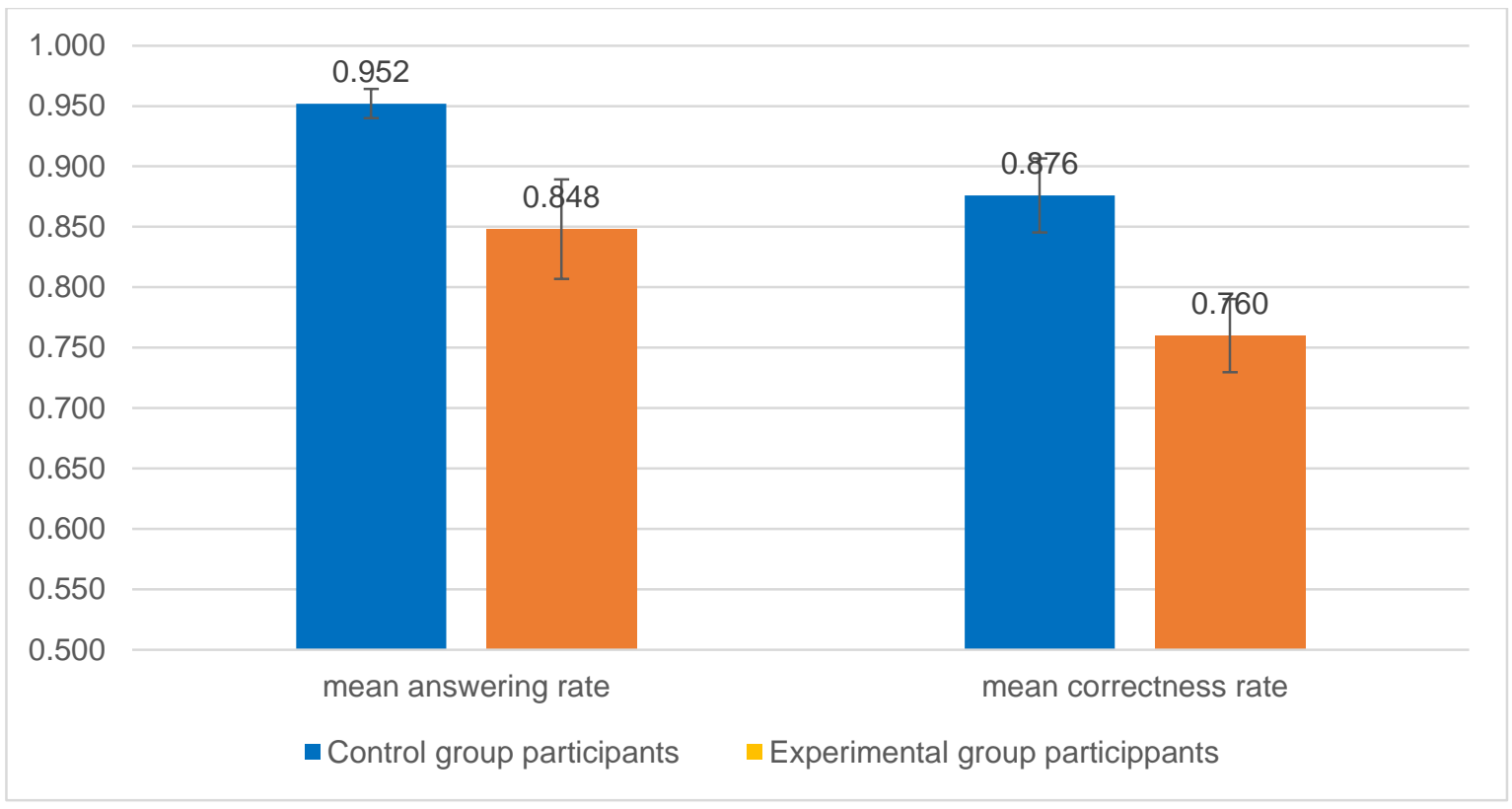

Fig. 4: Results on answering rate and correctness rate. Similar results are observed from the average time for verbalization, where the experimental group showed a slower response (2.19s) than the control group (2.44s) (fig. 5).

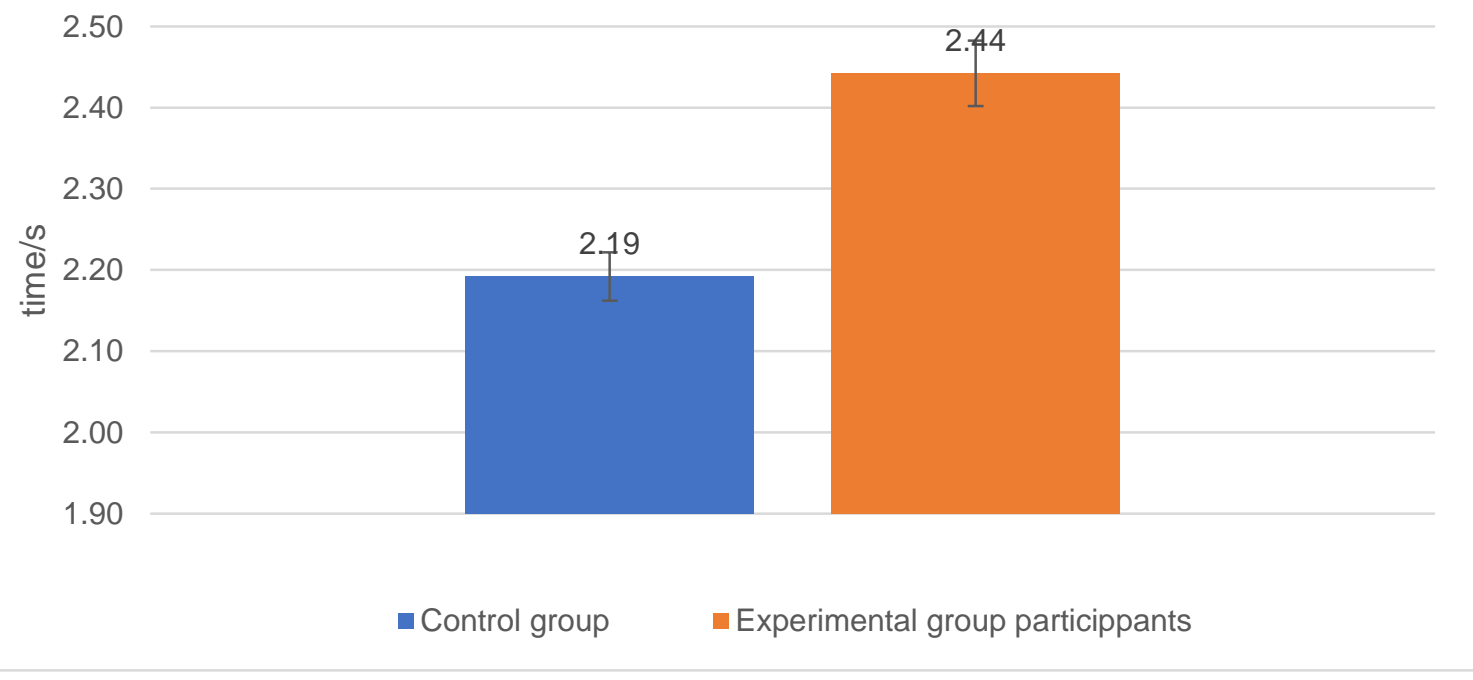

Fig. 5: Results on average verbalized response time.

We conducted an unpaired, two-tailed t-test in a follow-up analysis. The difference in answering rate and correctness rate were both significant ( $\mathrm{p}$-value $<.05$ ), suggesting that watching short videos reduced participants' accuracy in recognizing patterns by approximately $10 \%$. Short video viewing behaviors also significantly slower participants' response time (p-value $<.05)$.

\section{CONCLUSION}

Results from this experiment indicate that short video viewing can significantly reduce the answering rates in the following secondary task. Accuracy of participants'
VSTM for the stimulus suffers, and response time also increased after $25 \mathrm{~min}$ of short video viewing. Interestingly, subjects' performances in all three variances were poorer by approximately $10 \%$ (lower answering and correctness rate, longer response time). These results suggest that a daily average time of short video watching behaviors negatively affected VSTM.

Previous studies have shown that structural visual features are capable of interfering with VSTM. Specifically, the more difficult and structurally complex [11] or the more irrelevant [4] is the distractor, the more damage it causes to the VSTM. Simple distractors, on the other hand, have no effect or a boosting effect on VSTM. 
Lang and Thorson went on to suggest that this correlation is the outcome of viewers processing visual stimulus at a level close to, or exceeding, their processing capacity. Based on these prior studies, we can conclude that short videos possess a certain level of complexity that negatively impacts viewers' VSTM. However, since video contents used in this study were not strictly controlled and no orienting response was confirmed by measuring physiological data (e.g., heart rate), we cannot confidently identify perceptual limitation, attentional limitation, or processing capacity as the cause for the decrease in VSTM.

\section{LIMITATIONS AND FUTURE DIRECTIONS}

There are a few limitations within our research due to current constraints that can be corrected in further studies. First of all, the sample size of this experiment is limited with an unequal distribution of gender: among all 10 participants ( 8 females, 2 males), all male participants were randomly allocated to the experimental group, which may have affected the results. Secondly, while the experiment was initially designed to run directly on the PyschoPy interface with automatic measurement for response time, we had to take an alternative form to run online using zoom and manual recording of verbalized response time due to current social gathering restrictions and device limit. Finally, the video contents each participant viewed were not strictly instructed; while this may have better simulated their actual viewing habits, it also posed a possible confounding variant to this study.

As for future directions, we believe that there is more to explore from the experimental data. The level of damage on VSTM may differ among participants of different age groups or with different viewing habits, which can be further investigated once we acquire more varied subjects. Some neuroscientific studies (e.g., EEG and fMRI) may also provide useful evidence to support our conclusion and provide more insights into studies in this field.

\section{REFERENCES}

[1] TikTok. (2020, June 18). How TikTok recommends videos \#ForYou. Retrieved May 28, 2021, from TikTok website: https://newsroom.tiktok.com/en-us/how-tiktokrecommends- videos-for-you Anderson, D., \&

[2] Atkinson, R. C., \& Shiffrin, R. M. (1968). Human memory: A proposed system and its control processes. In K. W. Spence \& J. T. Spence (Eds.), Psychology of Learning and Motivation (Vol. 2, pp. 89-195). San Diego, CA: Elsevier.
[3] Averbach, E., \& Coriell, A. (1961). Short-term memory in vision. Bell System Technical Journal, 40, 309-328.

[4] Rademaker, R. L., Bloem, I. M., De Weerd, P., \& Sack, A. T. (2015). The impact of interference on short-term memory for visual orientation. Journal of Experimental Psychology. Human Perception and Performance, 41(6), 1650-1665.

[5] Reeves, B., Thorson, E., Rothschild, M., McDonald, D., Hirsch, J., \& Goldstein, R. (1985). Attention to Television: Intrastimulus Effects of Movement and Scene Changes on Alpha Variation Over Time. International Journal Of Neuroscience, 27(3-4), 241-255. doi: 10.3109/00207458509149770

[6] Levin, S. (1976). Young Children's Attention to "Sesame Street". Child Development, 47(3), 806. doi: $10.2307 / 1128198$

[7] Ohman, A. (1977). The orientation response, attention and learning: An information processing perspective. The Orienting Reflex In Humans, 443472.

[8] Lavie, N. (1995). Perceptual load as a necessary condition for selective attention. Journal of Experimental Psychology: Human Perception And Performance, 21(3), 451-468. doi: 10.1037/00961523.21.3.451

[9] Lang, A., Geiger, S., Strickwerda, M., \& Sumner, J. (1993). The Effects of Related and Unrelated Cuts on Television Viewers' Attention, Processing Capacity, and Memory. Communication Research, 20(1), 4-29. doi: 10.1177/009365093020001001

[10] Lang, A. (1991). Emotion, formal features, and memory for televised political advertisements. Television And Political Advertising, 1.

[11] Thorson, E., \& Lang, A. (1988). The effects of videographic complexity on memory for televised information. International Communication Association, New Orleans, LA.

[12] Newhagen, J., \& Reeves, B. (1991). Effects of compelling visual images on attention to and memory for new stories. Paper presented to the International Communication 